\title{
ON THE ARITHMETIC AND GEOMETRIC MEANS AND ON HÖLDER'S INEQUALITY
}

\section{H. KOBER}

1. The theorem on the arithmetic and geometric means can be sharpened; in consequence of this, Hölder's inequality can be sharpened also, and finally, Minkowski's inequality.

It is well known that the difference between the two means,

$$
D_{n}=\left(x_{1}+x_{2}+\cdots+x_{n}\right) / n-\left(x_{1} x_{2} \cdots x_{n}\right)^{1 / n},
$$

is the square of an irrational function for $n=2$, a sum of squares for $n>2$. For instance ${ }^{1}$

$$
D_{3}=\sum_{1 \leqq i<k \leqq 3}\left\{\left(x_{1}^{1 / 3}+x_{2}^{1 / 3}+x_{3}^{1 / 3}\right)^{1 / 2}\left(x_{i}^{1 / 3}-x_{k}^{1 / 3}\right) / 6^{1 / 2}\right\}^{2} .
$$

These representations are increasingly complicated with increasing $n$. But is there not a simple sum of squares that is comparable ${ }^{2}$ with $D_{n}$ in the Hardy-Littlewood-Pólya sense? In fact, $\sum\left(\left(x_{i}\right)^{1 / 2}-\left(x_{k}\right)^{1 / 2}\right)^{2}$ is, as will be shown.

Throughout this paper we suppose that not all the $x_{i}$ be equal; and we write $\sum u_{i k}$ or $\sum{ }^{n} u_{i k}$ when $1 \leqq i<k \leqq n$. We prove

Theorem 1. Let $n \geqq 2,0<q_{1} \leqq q_{2} \leqq \cdots \leqq q_{n}, \sum q_{i}=1, x_{i} \geqq 0$. Then

$$
\begin{aligned}
q_{1}(n-1)^{-1} & \leqq \frac{\Delta_{n}}{\sum\left(\left(x_{i}\right)^{1 / 2}-\left(x_{k}\right)^{1 / 2}\right)^{2}} \leqq q_{n} ; \\
\Delta_{n} & =\sum_{1}^{n} q_{i} x_{i}-x_{1}^{q_{1}} x_{2}^{q_{2}} \cdots x_{n}^{q_{n}} .
\end{aligned}
$$

If $q_{1}=q_{2}=\cdots=q_{m}$ for some $m, 1 \leqq m \leqq n$, then the lower bound $q_{1}(n-1)^{-1}$ is reached if and only if any one of the $x_{i}$ with $1 \leqq i \leqq m$ is positive and the other $n-1$ numbers $x_{i}$ vanish; except for the trivial case $n=2, q_{1}=q_{2}=1 / 2$. If $q_{1}<q_{2}=q_{3}=\cdots=q_{n}$ then the upper bound $q_{n}$ is attained if and only if $x_{1}=0$ and $x_{2}=x_{3}=\cdots=x_{n}>0$. If $q_{1}=q_{2}=\cdots=q_{n}=1 / n$ and $n \geqq 3$ then the upper bound $1 / n$ is

Received by the editors June 25, 1957 and, in revised form, October 20, 1957.

1 G. H. Hardy, J. E. Littlewood and G. Pólya, Inequalities, Cambridge, 1934, see $\$ 2.23$.

${ }^{2}$ Loc. cit., $\S \S 1.6,3.4$, where one-sided comparability is introduced. Here doublesided comparability of two functions $f$ and $g$ is considered, i.e. two inequalities $0 \leqq f \leqq c_{1} g$ and $0 \leqq g \leqq c_{2} f$, where $c_{1}$ and $c_{2}$ are positive constants. 
attained if and only if any one of the $x_{i}$ is zero and the other $n-1$ numbers $x_{i}$ are positive and equal.

Remarks. For (b), cf. Appendix.

(a) It is an open question whether $q_{n}$ is the least upper bound in the general case.

(b) When the point $\left\{x_{1}, x_{2}, \cdots, x_{n}\right\}$ in $n$-dimensional space $(n>2)$ tends to $\left\{x_{0}, x_{0}, \cdots, x_{0}\right\}$, where $x_{0}>0$, the fraction in (1.2) tends to a unique limit if, and only if, $q_{1}=q_{2}=\cdots=q_{n}=1 / n$. This limit is $2 n^{-2}$.

Concerning Hölder's inequality we deduce

Theorem 2. Let $0<q_{1} \leqq q_{2} \leqq \cdots \leqq q_{n}, \quad \sum q_{i}=1$ and $a_{i k} \geqq 0$; $i=1,2, \cdots, m ; j=1,2, \cdots, n ; m, n \geqq 2 ; a_{1 k}+a_{2 k}+\cdots+a_{m k}$ $=s_{k}>0$ for $k=1,2, \cdots, n$. Then $(c f .5 .1)$

$$
\sum_{i=1}^{m} a_{i 1}^{q_{1}} a_{i 2}^{q_{2}} \cdots a_{i n}^{q_{n}} \geqq \max \left\{0 ; s_{1}^{q_{1} s_{2}} \cdots s_{n}^{q_{n}}\left(1-q_{n} L\right)\right\},
$$

$$
\sum_{i=1}^{m} a_{i 1}^{q_{1} a_{i 2}} \cdots a_{i n}^{q_{2}} \leqq s_{1}^{q_{1}} s_{2}^{q_{2}} \cdots s_{n}^{q_{n}}\left(1-\frac{q_{1}}{n-1} L\right)
$$

where

$$
L=\sum_{i=1}^{m} \sum_{1 \leq j<k \leqq n}\left\{\left(\frac{a_{i j}}{s_{j}}\right)^{1 / 2}-\left(\frac{a_{i k}}{s_{k}}\right)^{1 / 2}\right\}^{2} .
$$

If $n=2$ and $q_{1}=q_{2}=1 / 2$, then trivially $\sum\left(a_{i 1} a_{i 2}\right)^{1 / 2}$ $=\left(s_{1} s_{2}\right)^{1 / 2}(1-L / 2)$.

The corresponding problem for Minkowski's inequality is more difficult, it plays a part in an investigation into arc length and surface area. One result is given in $\$ 6$.

2. On the lower bound in Theorem 1. First take $q_{1}<q_{2}$ and set $\Delta_{n}-\sum\left(\left(x_{i}\right)^{1 / 2}-\left(x_{k}\right)^{1 / 2}\right)^{2} q_{1}(n-1)^{-1}=d_{n}$. Then

$$
d_{n}=\sum_{i=2}^{n}\left(q_{i}-q_{1}\right) x_{i}+\left\{2 q_{1}(n-1)^{-1}\right\} \sum^{n}\left(x_{i} x_{k}\right)^{1 / 2}-x_{1}^{q_{1}} x_{2}^{q_{2}} \cdots x_{n}^{q_{n}} .
$$

But

$$
\sum\left(q_{i}-q_{1}\right)+\frac{n(n-1)}{2} 2 q_{1}(n-1)^{-1}=\left(1-n q_{1}\right)+n q_{1}=1 .
$$

Using the Theorem on the arithmetic and geometric means in its 
generalized form ${ }^{3}$ we deduce that $d_{n}>0$, unless

$x_{2}=x_{3}=\cdots=x_{n}=\left(x_{1} x_{2}\right)^{1 / 2}=\left(x_{1} x_{3}\right)^{1 / 2}=\cdots=\left(x_{2} x_{3}\right)^{1 / 2}=\cdots$,

i.e. unless $x_{1}>0$ and $x_{2}=x_{3}=\cdots=x_{n}=0$, since not all the $x_{i}$ are equal. In a similar way we deal with the cases $q_{1}=q_{2}<q_{3}, q_{1}=q_{2}$ $=q_{3}<q_{4}$, etc.

\section{Now we need a lemma.}

If $a_{i} \geqq 0, n>2$, not all the $a_{i}$ are equal and

$$
\text { (3.1) } g_{n}(a)=(n-2) \sum_{i=1}^{n} a_{i}+n\left(a_{1} a_{2} \cdots a_{n}\right)^{1 / n}-2 \sum^{n}\left(a_{i} a_{k}\right)^{1 / 2},
$$

then $g_{n}(a)>0$, unless one of the $a_{3}$ is zero and the others are positive and equal.

Without loss of generality we assume that $a_{1} \leqq a_{2} \leqq \cdots \leqq a_{n}$. Trivially $g_{2}(a) \equiv 0$. Suppose it be true that, for some $n>2, g_{n}(a) \geqq 0$ for all $\left(a_{1}, a_{2}, \cdots, a_{n}\right), a_{i} \geqq 0$. We consider $g_{n+1}(a)$ and set $a_{1}^{*}=a_{1}$, $a_{2}^{*}=a_{3}^{*}=\cdots=a_{n+1}^{*}=A=\left(a_{2} a_{3} \cdots a_{n+1}\right)^{1 / n}$. Then

$$
g_{n+1}\left(a^{*}\right)=(n-1) a_{1}+(n+1) a_{1}^{1 /(n+1)} A^{n /(n+1)}-2 n\left(a_{1} A\right)^{1 / 2} .
$$

If $a_{1}=0$ then $g_{n+1}\left(a^{*}\right)=0$ also. Let now $a_{1}>0$. Then

$$
\frac{g_{n+1}\left(a^{*}\right)}{2 n a_{1}^{1 /(n+1)}}=q_{1} a_{1}^{n /(n+1)}+q_{2} A^{n /(n+1)}-a_{1}^{q_{1} n /(n+1)} A^{q_{2} n /(n+1)},
$$

where $q_{1}=(n-1) /(2 n), q_{2}=(n+1) /(2 n) ; q_{1}+q_{2}=1$. Hence $g_{n+1}\left(a^{*}\right)$ $>0$, unless $a_{1}=A$, i.e. $a_{1}=a_{2}=\cdots=a_{n+1}$; which case, however, was e ccluded. Thus $g_{n+1}\left(a^{*}\right)>0$ unless $a_{1}=0$.

Now we consider $g_{n+1}(a)-g_{n+1}\left(a^{*}\right)=d_{n+1}$. We have

$$
\begin{aligned}
d_{n+1}= & (n-1) \sum_{i=2}^{n+1} a_{i}-2\left(a_{1}\right)^{1 / 2}\left(\sum_{i=2}^{n+1}\left(a_{i}\right)^{1 / 2}-n(A)^{1 / 2}\right) \\
& -2 \sum_{2 \leqq i<k \leqq n+1}\left(a_{i} a_{k}\right)^{1 / 2} \\
= & g_{n}\left(a_{2}, a_{3}, \cdots, a_{n+1}\right)+\sum_{i=2}^{n+1} a_{i} \\
& -2\left(a_{1}\right)^{1 / 2}\left(\sum_{i=2}^{n+1}\left(a_{i}\right)^{1 / 2}-n(A)^{1 / 2}\right)-n \prod_{i=2}^{n+1} a_{i}^{1 / n} .
\end{aligned}
$$

Since $g_{n}\left(a_{2}, a_{3}, \cdots, a_{n+1}\right) \geqq 0$ by assumption, $a_{1} \leqq A$ and $\left(a_{2} a_{3} \cdots a_{n+1}\right)^{1 / n}=A$,

${ }^{3}$ Loc. cit., Theorem 9, Inequality (2.5.2). 


$$
d_{n+1} \geqq \sum_{i=2}^{n+1} a_{i}-2(A)^{1 / 2} \sum_{i=2}^{n+1}\left(a_{i}\right)^{1 / 2}+n A=\sum_{i=2}^{n+1}\left(\left(a_{i}\right)^{1 / 2}-(A)^{1 / 2}\right)^{2} .
$$

This is positive, unless $a_{i}=A(i=2,3, \cdots, n+1)$, i.e. $a_{2}=a_{3}=\cdots$ $=a_{n+1}$. Combining the two results we complete the proof.

4. On the upper bound in (1.2). The denominator is

$$
\begin{aligned}
& \sum\left(\left(x_{i}\right)^{1 / 2}-\left(x_{k}\right)^{1 / 2}\right)^{2} \\
& =n D_{n}+\left\{(n-2) \sum x_{i}+n\left(x_{1} x_{2} \cdots x_{n}\right)^{1 / n}-2 \sum\left(x_{i} x_{k}\right)^{1 / 2}\right\} .
\end{aligned}
$$

By the lemma, the term in the curled brackets is positive, unless one of the $x_{i}$ is zero and the others are equal. Under this restriction, therefore, the fraction in (1.2) is smaller than $\Delta_{n}\left(n D_{n}\right)^{-1}$.

(i) When $q_{1}=q_{2}=\cdots=q_{n}=1 / n$ clearly $\Delta_{n}=D_{n}$, which completes the proof for the familiar case,

(ii) If not all the $q_{i}$ are equal, not all the differences $q_{n}-q_{i}$ vanish. Since

$$
D_{n}-\frac{\Delta_{n}}{n q_{n}}=\frac{1}{n q_{n}} \sum_{i=1}^{n-1}\left(q_{n}-q_{i}\right) x_{i}+\frac{1}{n q_{n}} \prod_{i=1}^{n} x_{i}^{q_{i}}-\prod_{i=1}^{n} x_{i}{ }^{1 / n}
$$

and

$$
\sum_{i=1}^{n-1} \frac{q_{n}-q_{i}}{n q_{n}}+\frac{1}{n q_{n}}=1 ; \quad \frac{q_{n}-q_{i}}{n q_{n}}+\frac{q_{i}}{n q_{n}}=\frac{1}{n},
$$

we can use the Theorem on the means. Hence $D_{n}-\Delta_{n} /\left(n q_{n}\right) \geqq 0$, therefore $\Delta_{n} /\left(n D_{n}\right) \leqq q_{n}$. Thus

$$
\Delta_{n} / \sum^{n}\left(\left(x_{i}\right)^{1 / 2}-\left(x_{k}\right)^{1 / 2}\right)^{2} \leqq q_{n} .
$$

If $q_{2}=q_{3}=\cdots=q_{n}>q_{1}$ the first term on the right in (4.2) reduces to $\left(q_{n}-q_{1}\right)\left(n q_{n}\right)^{-1} x_{1}$; therefore $D_{n}-\Delta_{n} /\left(n q_{n}\right)=0$ if, and only if, $x_{1}$ $=x_{1}^{q_{1}} x_{2}^{q_{2}} \cdots x_{n}^{q_{n}}$. Combining this with the above condition resulting from the lemma we deduce that $x_{1}=0, x_{2}=x_{3}=\cdots=x_{n}>0$, which completes the proof.

5. On Hölder's inequality. We employ a well-known argument. ${ }^{4}$ Set $a_{i k} / s_{k}=b_{i k}$; then $b_{1 k}+b_{2 k}+\cdots+b_{m k}=1$ for each $k$. Now

$$
1-\sum_{i=1}^{m} b_{i 1}^{q_{1}} b_{i 2}^{q_{2}} \cdots b_{i n}^{q_{n}}=\sum_{i=1}^{m}\left(q_{1} b_{i 1}+q_{2} b_{i 2}+\cdots-q_{n} b_{i n}+\prod_{k=1}^{n} b_{i k}^{q_{k}}\right) .
$$

We can apply Theorem 1 to each of the expressions in brackets. Thus

‘ Loc. cit. \$2.7, proof (ii) of Hölder's inequality. 


$$
\begin{aligned}
& \frac{q_{1}}{n-1} \sum_{i=1}^{m} \sum_{1 \leq j<k \leqq n}\left(\left(b_{i j}\right)^{1 / 2}-\left(b_{i k}\right)^{1 / 2}\right)^{2} \\
& \leqq 1-\sum_{i=1}^{m} \prod_{k=1}^{n} b_{i k}^{q_{k}} \leqq \min \left\{1, q_{n} \sum_{i=1}^{m} \sum_{1 \leq j<k \leq n}\left(\left(b_{i j}\right)^{1 / 2}-\left(b_{i k}\right)^{1 / 2}\right)^{2}\right\},
\end{aligned}
$$

which is equivalent to the result required. Clearly it implies that $\sum b_{i 1}^{q_{1}} b_{i 2}^{q_{2}} \cdots b_{i n}^{q_{n}}=1$ if, and only if, for each $i, j$ and $k$ the numbers $b_{i j}$ and $b_{i k}$ are equal.

Henceforward we exclude this. In special cases certainly equality in (5.1) can be attained. For $n=2, m \geqq 2$, see $\S 1$. When $m=n$ and $q_{1}=q_{2}=\cdots=q_{n}=1 / n$, for instance, there is equality on the left if and only if, for some permutation $\kappa, \lambda, \cdots, \omega$ of the numbers $1,2, \cdots, n, b_{1 \kappa}=b_{2 \lambda}=\cdots=b_{m \omega}=1$; equality on the right if and only if $b_{1 \kappa}=b_{2 \lambda}=\cdots=b_{m \omega}=0$ while all the other $b_{i k}$ are positive and equal (i.e., $=1 /(m-1))$.

\section{A general, yet preliminary, result on Minkowski's inequality.}

THEOREM 3 . Let $m \geqq 2, \quad n \geqq 2, \quad r>1, \quad r^{\prime}=r(r-1)^{-1}, \quad a_{i k} \geqq 0, \quad \rho^{r}$ $=\sum_{i=1}^{m} a_{i k}^{r}>0$,

$$
\begin{aligned}
S^{r} & =\sum_{i=1}^{m}\left(\sum_{k=1}^{n} a_{i k}\right)^{r} \\
L_{k} & =\sum_{i=1}^{m}\left\{\left(\frac{a_{i 1}+\cdots+a_{i n}}{S}\right)^{r / 2}-\left(\frac{a_{i k}}{\rho_{k}}\right)^{r / 2}\right\}^{2} .
\end{aligned}
$$

Then

$$
\frac{1}{\max \left(r, r^{\prime}\right)} \sum_{k=1}^{n} \rho_{k} L_{k} \leqq \sum_{k=1}^{n} \rho_{k}-S \leqq \frac{1}{\min \left(r, r^{\prime}\right)} \sum_{k=1}^{n} \rho_{k} L_{k} .
$$

Clearly there is equality whenever $r=2$; and $\sum_{k=1}^{n} \rho_{k}>S$, unless $a_{i k} / \rho_{k}=a_{i l} / \rho_{l}$ for any $i, k, l$, i.e. unless the $a_{\imath 1}, a_{i 2}, \cdots$ are proportional.

The proof is based on a well-known argument. ${ }^{5}$ If $\sigma_{i}=\sum_{k=1}^{n} a_{i k}$, $b_{i k}=a_{i k}^{r}$, then

$$
\begin{gathered}
S=S^{1-r}\left\{\sum_{i=1}^{m} a_{i 1} \sigma_{i}^{r-1}+\sum_{i=1}^{m} a_{i 2} \sigma_{i}^{r-1}+\cdots+\sum_{i=1}^{m} a_{i n} \sigma_{i}^{r-1}\right\}, \\
a_{i k} \sigma_{i}^{r-1}=b_{i k}^{1 / r}\left(\sigma_{i}^{r}\right)^{1 / r^{\prime}} .
\end{gathered}
$$

We fix $k(=1$, or $2, \cdots, n)$ and use Theorem 2. If $r>r^{\prime}$ let $q_{1}=1 / r$,

\footnotetext{
s Loc. cit., \$2.11, first proof of Minkowski's inequality.
} 
$q_{2}=1 / r^{\prime} ; s_{1}=\sum_{i=1}^{m} b_{i k}=\rho_{k}^{r}, s_{2}=\sum_{i=1}^{m} \sigma_{i}^{r}=S^{r} ;$ we deduce that

$$
\rho_{k} S^{r-1}\left(1-q_{2} L_{k}\right) \leqq \sum_{i=1}^{m} b_{i k}^{1 / r}\left(\sigma_{i}^{r}\right)^{1 / r^{\prime}} \leqq \rho_{k} S^{r-1}\left(1-q_{1} L_{k}\right) .
$$

If $r<r^{\prime}$ interchange $q_{1}$ and $q_{2}$, and $s_{1}$ and $s_{2}$ also. Combining these results we can complete the proof. A theorem which is more suitable for applications will be given in another paper.

Appendix. Added on October 10, 1957. We prove the Remark (b) to Theorem 1 ; and in addition, two results: if $n=2, q_{1} \gtrless q_{2}$, the fraction in (1.2) tends to a unique limit when $\left(x_{1}-x_{0}\right)^{2}+\left(x_{2}-x_{0}\right)^{2} \rightarrow 0$, where $x_{0}>0$ is fixed; for $n \geqq 2$, there is never a unique limit when $x_{0}=0$, except for the trivial case $n=2, q_{1}=q_{2}=1 / 2$.

We set $U_{n}(x)=\sum\left(\left(x_{i}\right)^{1 / 2}-\left(x_{k}\right)^{1 / 2}\right)^{2}(1 \leqq i<k \leqq n)$ and take $\sum_{i}\left(x_{i}-x_{0}\right)^{2} \rightarrow 0$. We observe that not all the $x_{i}$ are equal.

I. Let $n>2, q_{1}<q_{n}$. If $x_{2}=x_{3}=\cdots=x_{n}=0, x_{1}=x_{0}+\epsilon(\epsilon \gtrless 0)$,

$$
\Delta_{n}(x) /\left(U_{n}(x) \rightarrow 2(n-1)^{-1} q_{1}\left(1-q_{1}\right)\right.
$$

by the binomial series. If, however, $x_{1}=\cdots=x_{n-1}=0, x_{n}=x_{0}+\epsilon$, the limit is $2(n-1)^{-1} q_{n}\left(1-q_{n}\right)>2(n-1)^{-1} q_{1}\left(1-q_{1}\right)$. Thus there is no unique limit.

II. Let $n>2, q_{1}=q_{2}=\cdots=q_{n}=1 / n$. We need only show the existence of a unique limit. Then (1.1) implies that the latter is $2 n^{-2}$. Set $x_{i}=X_{i}^{2 n}(i=1,2, \cdots, n), x_{0}=A^{2 n}$. Then

$$
U_{n}\left(X^{2 n}\right)=\sum_{1 \leqq i<k \leqq n}\left(X_{i}-X_{k}\right)^{2} P_{i k} ; \quad P_{i k}=\left(\sum_{j=0}^{n-1} X_{i}^{n-1-j} X_{k}^{j}\right)^{2} .
$$

Hence, given a sufficiently small $\epsilon>0$, there is a $\delta$ such that

$$
0<n^{2} A^{2 n-2}-\epsilon<P_{i k}<n^{2} A^{2 n-2}+\epsilon, \quad\left\{\sum_{i}\left(X_{i}-A\right)^{2}<\delta\right\} .
$$

Now it follows from the Hurwitz-Muirhead identity ${ }^{6}$ that the polynomial $n \Delta_{n}\left(X^{2 n}\right)=\sum_{i} X_{i}^{2 n}-n X_{1}^{2} X_{2}^{2} \cdots X_{n}^{2}$ is representable in the form

$$
n \Delta_{n}\left(X^{2 n}\right)=\sum_{1 \leqq i<k \leqq n}\left(X_{i}-X_{k}\right)^{2} Q_{i k},
$$

where the poynomials $Q_{i k} \equiv Q_{k i}$ are of degree $2 n-2$ and are homogeneous in the variables $X_{1}, X_{2}, \cdots, X_{n}$, with all their coefficients positive. Let $K$ be the sum of the coefficients of $Q_{i k}$. Since $Q_{j l}$ may

6 Loc. cit., $\$ 2.23(2.23 .1)$ and $\$ 2.21(1)$. 
be derived from $Q_{i k}$ by interchanging $X_{i}$ with $X_{j}$ and $X_{k}$ with $X_{l}$, $K$ is independent of $i, k$. Thus, for some $\delta>0$,

$$
0<K A^{2 n-2}-\epsilon<Q_{i k}<K A^{2 n-2}+\epsilon, \quad\left\{\sum_{i}\left(X_{i}-A\right)^{2}<\delta\right\} .
$$

By (1.4) and (1.5),

$$
\frac{K A^{2 n-2}-\epsilon}{n^{2} A^{2 n-2}+\epsilon}<\frac{n \Delta_{n}}{U_{n}}<\frac{K A^{2 n-2}+\epsilon}{n^{2} A^{2 n-2}-\epsilon} .
$$

As $\epsilon \rightarrow 0, \Delta_{n} / U_{n} \rightarrow K n^{-3}$; which shows the existence of the limit.

1II. The case $n=2$. We show that the function

$$
f(x, y ; p)=\left(p x+q y-x^{p} y^{q}\right) /\left(x^{1 / 2}-y^{1 / 2}\right)^{2} \quad(0<p<p+q=1)
$$

tends to $2 p q$ as $(x-a)^{2}+(y-a)^{2} \rightarrow 0$, where $a>0$ is fixed. If $p$ is rational, this follows readily ${ }^{7}$ from the preceding result.

Let now $p$ be irrational and let $\left\{P_{m}\right\}$ and $\left\{p_{m}\right\}(m=1,2, \cdots)$ be sequences of rational numbers tending to $p$ and such that $P_{m}>p>p_{m}$. Then $Q_{m} \rightarrow q, q_{m} \rightarrow q(m \rightarrow \infty)$, where $Q_{m}=1-P_{m}, q_{m}=1-p_{m}$, and

$$
\lim _{(x, y) \rightarrow(a, a)} f\left(x, y ; P_{m}\right)=2 P_{m} Q_{m} ; \quad \lim _{(x, y) \rightarrow(a, a)} f\left(x, y ; p_{m}\right)=2 p_{m} q_{m} .
$$

(i) Let $x>y>0, x / y=u(>1)$, and consider the function $g(s)$ $=s x+(1-s) y-x^{s} y^{1-8}$ for fixed $x, y$. Suppose first that $p<1 / 2$. We may take $P_{m}<1 / 2(m=1,2, \cdots)$ and $s<1 / 2$. Then $2 p_{m} q_{m}<2 p q$ $<2 P_{m} Q_{m}$,

$$
\begin{aligned}
\frac{g^{\prime}(s)}{y} & =u-1-u^{s} \log u>u-1-u^{1 / 2} \log u \\
& =u^{1 / 2} \int_{1}^{u}\left(v^{1 / 4}-v^{-1 / 4}\right)^{2} \frac{d v}{2 v} \\
& >0 .
\end{aligned}
$$

Hence $g\left(P_{m}\right)>g(p)>g\left(p_{n}\right)$; as $g\left(p_{m}\right)=\left(x^{1 / 2}-y^{1 / 2}\right)^{2} f\left(p_{m}\right)$, by $(1.6)$

$$
\text { (1.7) } 2 p_{m} q_{m} \leqq \liminf _{(x, y) \rightarrow(a, a)} f(x, y ; p) \leqq \limsup _{(x, y) \rightarrow(a, a)} f(x, y ; p) \leqq 2 P_{m} Q_{m} \text {. }
$$

Let now $p>1 / 2$. Then we may suppose that, for some constant $c>1 / 2, c<p_{m}<p<P_{m}<1(m=1,2, \cdots)$. Set $u_{0}=\left\{c(1-c)^{-1}\right\}^{1 /(1-c)}$. Then $u_{0}>1$,

${ }^{7}$ Loc. cit., cf. $\$ 2.2$ and the proof of (2.5.1). 


$$
\begin{aligned}
\frac{g^{\prime}(s)}{y u^{c}} & \leqq \frac{u-1-u^{c} \log u}{u^{c}}=\int_{1}^{u}\left\{(1-c) v+c-v^{c}\right\} \frac{d v}{v^{1+c}} \\
& =c \int_{1}^{u} \frac{d v}{v^{1+c}} \int_{1}^{v}\left(\frac{1}{u_{0}^{1-c}}-\frac{1}{z^{1-c}}\right) d z ;
\end{aligned}
$$

which is certainly negative for $1<u<u_{0}$. Since $x \rightarrow a$ and $y \rightarrow a$, we may take $u$, i.e. $x / y,<u_{0}$. Hence $f\left(x, y ; P_{m}\right)<f(x, y ; p)<f\left(x, y ; p_{m}\right)$, and we obtain an inequality similar to (1.7), with $2 p_{m} q_{m}$ and $2 P_{m} Q_{m}$ interchanged. Taking $m \rightarrow \infty$, we have

$$
\lim _{(x, y) \rightarrow(a, a)} f(x, y ; p)=2 p q .
$$

(ii) Let now $y>x$. We arrive at the same result when observing that $f(x, y ; p)=f(y, x ; q) \rightarrow 2 q p$; thus we can complete the proof.

IV. The last statement is deduced by an argument similar to that used in I. Thus all the assertions are true.

BirminghaM, ENGLAND 